\title{
The Question of Ethics in Nanomedicine
}

\author{
Abdolhassan Kazemi, Maryam Majidinia* and Ali Akbar Jamali
}

Medical faculty, Tabriz University of Medical Sciences, Iran

*Corresponding author: Maryam Majidinia, Medical faculty, Tabriz University of Medical Sciences, Iran, Tel: 0989360488846; E-mail: Majidinia_m@yahoo.com

Rec date: Jun 07, 2014, Acc date: Aug 13, 2014, Pub date: Aug 16, 2014

Copyright: () 2014 Kazemi A, et al. This is an open-access article distributed under the terms of the Creative Commons Attribution License, which permits unrestricted use, distribution, and reproduction in any medium, provided the original author and source are credited.

\begin{abstract}
Lately, nanotechnology and nanomedicine have achieved tremendous interest and funding, from several entities and directions. Nanomedicine demonstrates great promise for development of medical diagnosis, treatment, and prevention; it also raises various ethical concerns however, there has not been so much study and discussion about social and ethical issues. Nanomaterials are extremely reactive in biological systems owing to the large surface area. Although the benefits of nanomaterials are apparent, there are some studies which show the potential risk to biological systems. Therefore, the rapid progress of nanomedicine and its potentially profound social and environmental impacts is indicative of how the arisen problems could be dangerous and already there are signs that some challenging ethical issues could be raised. In this paper we will examine more recent developments in debates on nanomedicine ethics.
\end{abstract}

Keywords: Ethics; Nanomedicine; Nanotechnology; Toxicity

\section{Introduction}

Recently, nanomedicine has been considered as developing scientific research area. Most of the existing debates surrounding ethical issues in nanomedicine are consequent of the past technologies such as biotechnology and information technology [1]. Debates also exist over whether nanomedicine has any unique ethical issues or the ethical issues of past technologies apply to nanoscience [2]. Researchers have widely written about probable ethical scenarios that might coincide with the development of nanoscience [3]. Other studies conducted in the area of nanotoxicology have suggested risks, including side effects of nanoparticles on humans, fish and the environment [3-6].

Pharmaceutical and biotechnology companies and government agencies have begun investigating and experimentation of various nanotechnology applications in nanomedicine. Some of nanoparticlebased therapies or imaging devices are presently being used in clinical trials and awaiting clinical trials, or have already been accepted by the Food and Drug Administration (FDA). Several nanomedicine have been approved for cancer treatments or are currently being experienced on human subjects [7]. The efficient targeted therapeutic prospective for nanomedicine can generate the potential side effects, if the precision becomes wrongly applied or if it has more than just the desired effect. Bottom-up manufacture from the molecular level, based either on mimicking biology or on incorporating components from living systems, raises issues about hybrid devices that interface human and machine, and also about priorities [8].

This paper presents the perspectives of practitioners about ethical issues related to nanomedicine. Many industrial and developing countries have allocated significant amounts of funding for nanotechnological research. Until now, however, there has been little formal study, debating, or thinking concerning the social and ethical issues related to nanomedicine all over the world. Therefore, it seems that we need to consider assessment for ethics training and scholarship in nanomedicine as well as preliminary proposals for research ethics and medical ethics training for scientists and clinicians as well as for empirical research in nanoethics.

\section{Nanomedicine}

New medical techniques and novel scientific discoveries bring a lot of critical questions relating to the role of human dignity in medical research and in the society of the future [9]. Nanomedicine is estimated to improve human capabilities and properties and assures the ability of health care professionals to diagnose, treat, and share medical information nearly instantly [10]. Nanomedicine includes the application of nanoparticles and nanodevices for diagnostics, targeted drug delivery in the human body, the production of new therapeutic materials such as nanorobots or nanoprotheses and biosensing [10-12].

The ability to monitor and intervene at a cellular or molecular level may fall foul of the complexity of the system. Nanomedicine may enable rapid readouts of our whole genome or of our body's levels of everything imaginable. Knowing all the information that nanomedicine could provide, is not necessarily a good thing [8]. As nanomedicine emerges as a vital and robust tool for the practice of medicine, it is becoming apparent that we need for ethics scholarship and training in nanomedicine.

\section{Ethical issues in nanomedicine}

Since the science and technology of nanomedicine is quickly expanding, ethical and law issues should be taken into consideration. Several nanoethicists have lately identified the need for 'better' ethics of emerging technologies and believed that researchers should consider ethical reflection as part and parcel of the research and development processes and should be transferred to nanomedicine [13]. Therefore, it is essential to proactively address the ethical, social and regulatory aspects of nanomedicine in order to diminish its side effects on the environment and public health and also to avoid a public reaction $[11,14]$. Also a recognition of the ethical issues involved may be helpful to decision makers, particularly employers, workers, investors, and health authorities. Because the goal of occupational 
safety and health is the prevention of disease in workers, the situations that have ethical implications that most affect workers have been identified [15]. Consequently, nanomedicine is poised to add a profound and complex set of ethical and societal questions [16].

Currently, the ethical considerations involved in nanomedicine are related to risk assessment in general, somatic-cell versus germline-cell therapy, the enhancement of human capabilities, risk management of engineered nanomaterials, research into human embryonic stem cells and the toxicity of nanoparticles and nanomedicine, uncontrolled function and self-assembly of nanoparticles [12,17]. For instance, recently, the identification of cytotoxicity of nanoparticles toward mammalian gremline stems cells has aroused great concern over the biosafety of nanomaterials [18]. In their study, they used a cell line with spermatogonial stem cell characteristics to test in vitro toxicity of several types of nanoparticles. In cell culture tests, the surfaces modified nanoparticles were internalized into platelet and fibroblast cells in a short period of incubation without harming the cells [19]. Toxic effects of nanoparticles on health despite the many benefits of nanotechnology, some studies indicate that certain nanoparticles may cause adverse effects because of their small size and unique properties $[20,21]$. Indeed, their size makes them highly mobile in both the human body and the environment. Nanomaterials can enter human tissues through several ports via the lungs after inhalation [22], through the digestive system, and possibly through the skin [23]. Systemic distribution of nanoparticles has been demonstrated after inhalation and oral uptake [24], and nanoparticles have been found to cross the blood brain barrier, reaching the olfactory bulb and the cerebellum [25]. Many of the artificially manufactured nanoparticles are made of non-biodegradable pollutants, such as carbon black and metals, and the long-term behaviour of such substances is not known. Silver has potentially toxic effects on human health and it can enter into the human body through various portals. Previous literature [26] indicated that $\mathrm{Ag}^{+}$causes early changes in the permeability of the cell membrane to $\mathrm{K}^{+}$and then to $\mathrm{Na}^{+}$at concentrations that don't limit $\mathrm{Na}$ ${ }^{+}, \mathrm{K}^{+}$-ATP activity. As per the existing literature [27], it has been demonstrated that silver nanoparticles show intensive toxic effects on the proliferation and cytokine expression by peripheral blood mononuclear cells (PMBCs) [28]. Silver does not only cause dermal and cosmetic toxic effects, but also it causes death in animals. Nanoparticles, such as silver nanoparticles, are showing severe toxic effects on the male reproductive system. The identified research suggests that nanoparticles cross the blood-testes barrier and are deposited in the testes, and that there is potential for adverse effects on sperm cells [29]. In the literature of Burd et al., it was demonstrated that commercially available silver-based dressings are also showing potential cytotoxic effects. The cytotoxicity correlated with the silver released from the dressings as measured by the silver concentration in the culture medium [30]. Cadmium is a recognized toxicant that has been classified as a probable human carcinogen. It is a heavy metal that has the potential to cause lysosomal damage and DNA breakage in mammalian hepatocytes [31] and many other cells and tissues [32]. Cadmium also disrupts mitochondrial function both in vivo [33] and in vitro [34], and promotes apoptosis [35]. In the testis, cadmium induces lysosomal damage in testicular Sertoli cells [36], but its main toxic effects appear in germ cells but not in somatic cells [37]. Subsequently, a decrease in the number of spermatogonia in relation to the time of exposure was observed, followed by a decrease in the number of spermatocytes and, ultimately, sperm cells. In humans, male infertility is strongly linked to cadmium exposure, but is rather due to a failure of the acrosomal reaction in sperm cells [38]. Although molybdenum in soluble form is considered to be a mildly toxic substance, it did not significantly affect the metabolic activity or the membrane integrity of the C18-4 cells. Interestingly, whereas the effect on mitochondrial function is mild, molybdenum nanoparticles seem to promote some plasma membrane leakage at very low concentrations $(5 \mu \mathrm{g} / \mathrm{ml}$ and $10 \mu \mathrm{g} / \mathrm{ml})$. The same pattern of toxicity is shown for aluminium nanoparticles; however, the morphology of the cells did not change, indicating that at these concentrations apoptosis still occurs. It is known that extreme and visible membrane damage occurs only in the late stages of apoptosis. It is shown that Aluminium nanoparticles (Al-NP) exhibit greater toxicity and more significantly diminishes the phagocytotic ability of macrophages after $24 \mathrm{~h}$ of exposure when compared to $\mathrm{Al}_{2} \mathrm{O}_{3}-\mathrm{NP}$.

Ethical investigation should also consider some of the reductionistic implications of engineering models and metaphors integral to nanomedicine, as well as application of nanomedicine for non-medical purposes, like human enhancement. Most of these challenges concern rate-limiting steps in nanomedical research, and they should be significantly featured in developing nanomedicine proposals [39].

Even though in vivo animal experiments and ex vivo laboratory analyses can increase our understanding of the interaction of engineered nanomaterials in biological systems, they cannot eliminate all of the uncertainty surrounding the exposure of a human subject to nanomedicine products in clinical trials. Also nanomedicine cytotoxicity and mutagenicity investigations are still in the early stage and even sometime contradictory. Some reports show that the advanced nanomaterials may inadvertently impose undesired intrinsic biological impacts which need to be considered $[6,12,14]$. Furthermore, as the use of engineered nanomaterials in nanomedicine increases, questions of social justice, access to healthcare and the use of nanotechnology for physical enhancement become increasingly important $[14,40]$.

\section{Conclusion}

This brief survey has mapped some of the ethical and social topography of the uncharted lands that nanotechnology may be offered to medicine, and posed many questions for debate. How, as societies, we seek answers as these technologies emerge will be of crucial importance. Scientists and clinicians in nanomedicine owe it to themselves and to the wider society to reflect on them, so that the right benefits may be achieved with justice, while retaining our humanity in all its rich diversity. Nanotechnology supporters think that it has the potential to transform our lives dramatically, whereas opponents of nanotechnology fear that self-replicating "nanobots" could escape from laboratories and diminish all life on earth. A focus on the ethical, legal, and societal implications of the technology, and especially respect for human nature, is therefore sensible. But it is not sufficient to fund research on societal questions; respect for the human condition must frame the development of nanomedicine. This paper demonstrated that none of the ethical questions surrounding nanomedicine are new or unique, and would hold true for any new medical device or medicine that was being evaluated.

\section{Acknowledgement}

The financial support of the Iranian Nanotechnology Initiative Council and Research Center for Pharmaceutical Nanotechnology, Tabriz University of Medical Sciences are gratefully acknowledged. 
Citation: Kazemi A, Majidinia M, Jamali AA (2014) The Question of Ethics in Nanomedicine. J Clinic Res Bioeth 5: 193. doi: $10.4172 / 2155-9627.1000193$

Page 3 of 3

\section{References}

1. Jamison A (2009) Can Nanotechnology Be Just? On Nanotechnology and the Emerging Movement for Global Justice. Nanoethics 3: 129-136.

2. Nordmann A, Rip A (2009) Mind the gap revisited. Nat Nanotechnol 4: 273-274.

3. Patra D, Haribabu E, McComas KA (2010) Perceptions of Nano Ethics among Practitioners in a Developing Country: A Case of India. Nanoethics 4: 67-75.

4. Gilbert N (2009) Nanoparticle safety in doubt. Nature 460: 937.

5. Sandler R (2009) Nanomedicine and nanomedical ethics. Am J Bioeth 9: 16-17.

6. Ezzati Nazhad Dolatabadi J, Omidi Y, Losic D (2011) Carbon Nanotubes as an Advanced Drug and Gene Delivery Nanosystem. Curr Nanosci, In press

7. Resnik DB, Tinkle SS (2007) Ethical issues in clinical trials involving nanomedicine. Contemp Clin Trials 28: 433-441.

8. Bruce D (2006) The question of ethics. Nanotoday 1: 6-7.

9. ZyciÅski J (2006) Ethics in medical technologies: the Roman Catholic viewpoint. J Clin Neurosci 13: 518-523.

10. Kuiken T (2010) Nanomedicine and ethics: is there anything new or unique? Wiley Interdiscip Rev Nanomed Nanobiotechnol.

11. Lenk C, Biller-Andorno N (2007) Nanomedicine-emerging or reemerging ethical issues? A discussion of four ethical themes. Med Health Care Philos 10: 173-184.

12. Ezzati Nazhad Dolatabadi J, Mashinchian O, Ayoubi B, Jamali AA, Mobed A, et al. (2010) Optical and Electrochemical DNA Nanobiosensors. Trend Anal Chem, In press.

13. Schuurbiers D, Sleenhoff S, Jacobs JF, Osseweijer P (2009) Multidisciplinary Engagement with Nanoethics Through Education-The Nanobio-RAISE Advanced Courses as a Case Study and Model. Nanoethics 3: 197-211.

14. Resnik DB, Tinkle SS (2007) Ethics in nanomedicine. Nanomedicine (Lond) 2: 345-350.

15. Schulte PA, Salamanca-Buentello F (2007) Ethical and scientific issues of nanotechnology in the workplace. Cien Saude Colet 12: 1319-1332.

16. Bawa R, Johnson S (2007) The ethical dimensions of nanomedicine. Med Clin North Am 91: 881-887.

17. Ebbesen M, Jensen TG (2006) Nanomedicine: techniques, potentials, and ethical implications. J Biomed Biotechnol 2006: 51516.

18. Service RF (2003) American Chemical Society meeting. Nanomaterials show signs of toxicity. Science 300: 243.

19. Hoet PH, Brüske-Hohlfeld I, Salata OV (2004) Nanoparticles - known and unknown health risks. J Nanobiotechnology 2: 12.

20. Oberdörster G (2001) Pulmonary effects of inhaled ultrafine particles. Int Arch Occup Environ Health 74: 1-8.

21. Jani P, Halbert GW, Langridge J, Florence AT (1990) Nanoparticle uptake by the rat gastrointestinal mucosa: quantitation and particle size dependency. J Pharm Pharmacol 42: 821-826.

22. Lademann J, Weigmann H, Rickmeyer C, Barthelmes H, Schaefer H, et al. (1999) Penetration of titanium dioxide microparticles in a sunscreen formulation into the horny layer and the follicular orifice. Skin Pharmacol Appl Skin Physiol 12: 247-256.
23. Oberdörster G, Sharp Z, Atudorei V, Elder A, Gelein R, et al. (2002) Extrapulmonary translocation of ultrafine carbon particles following whole-body inhalation exposure of rats. J Toxicol Environ Health A 65: 1531-1543.

24. Borm PJ, Kreyling W (2004) Toxicological hazards of inhaled nanoparticles--potential implications for drug delivery. J Nanosci Nanotechnol 4: 521-531.

25. Kone BC, Kaleta M, Gullans SR (1988) Silver ion (Ag+)-induced increases in cell membrane $\mathrm{K}+$ and $\mathrm{Na}+$ permeability in the renal proximal tubule: reversal by thiol reagents. J Membr Biol 102: 11-19.

26. Seung-heon S, Mitikyung Y, Jeung-kyu K (2006) The effects of nanosilver on the proliferation and cytokine production in peripheral blood mononuclear cells. Jpn. J. Rhinol. 45: 269-273.

27. McAuliffe ME, Perry MJ (2007) Are nanoparticles potential male reproductive toxicants? A literature review. Nanotoxicology 1: 204-210.

28. Burd A, Kwok CH, Hung SC, Chan HS, Gu H, et al. (2007) A comparative study of the cytotoxicity of silver-based dressings in monolayer cell, tissue explant, and animal models. Wound Repair Regen 15: 94-104.

29. Fotakis G, Cemeli E, Anderson D, Timbrell JA (2005) Cadmium chloride-induced DNA and lysosomal damage in a hepatoma cell line. Toxicol In Vitro 19: 481-489.

30. Satoh M, Koyama H, Kaji T, Kito H, Tohyama C (2002) Perspectives on cadmium toxicity research. Tohoku J Exp Med 196: 23-32.

31. Belyaeva EA, Glazunov VV, Korotkov SM (2002) Cyclosporin Asensitive permeability transition pore is involved in $\mathrm{Cd}(2+)$-induced dysfunction of isolated rat liver mitochondria: doubts no more. Arch Biochem Biophys 405: 252-264.

32. Pourahmad J, O'Brien PJ (2000) A comparison of hepatocyte cytotoxic mechanisms for $\mathrm{Cu} 2+$ and $\mathrm{Cd} 2+$. Toxicology 143: 263-273.

33. Pulido MD, Parrish AR (2003) Metal-induced apoptosis: mechanisms. Mutat Res 533: 227-241.

34. Boscolo P, Sacchettoni-Logroscino G, Ranelletti FO, Gioia A, Carmignani M (1985) Effects of long-term cadmium exposure on the testis of rabbits: ultrastructural study. Toxicol Lett 24: 145-149.

35. Aoyagi T, Ishikawa H, Miyaji K, Hayakawa K, Hata M (2002) Cadmiuminduced testicular damage in a rat model of subchronic intoxication. Reprod. Med. Biol. 1: 59-63.

36. Benoff S, Jacob A, Hurley IR (2000) Male infertility and environmental exposure to lead and cadmium. Hum Reprod Update 6: 107-121.

37. Xia T, Kovochich M, Brant J, Hotze M, Sempf J, et al. (2006) Comparison of the abilities of ambient and manufactured nanoparticles to induce cellular toxicity according to an oxidative stress paradigm. Nano Lett 6: 1794-1807.

38. Gopinath P, Gogoi SK, Chattopadhyay A, Ghosh SS (2008) Implications of silver nanoparticle induced cell apoptosis for in vitro gene therapy. Nanotechnology 19: 075104.

39. Takenaka S, Karg E, Roth C, Schulz H, Ziesenis A, et al. (2001) Pulmonary and systemic distribution of inhaled ultrafine silver particles in rats. Environ Health Perspect 109 Suppl 4: 547-551.

40. Khushf G (2007) Upstream ethics in nanomedicine: a call for research. Nanomedicine (Lond) 2: 511-521. 